\title{
Epilepsy Detection Using EEG with Different Time Frames
}

\author{
Jianguo Liu \\ Department of Mathematics, University of North Texas Denton, TX 76203, USA \\ jgliu@unt.edu
}

\begin{abstract}
The electroencephalogram (EEG) signal is widely used in clinical to investigate brain disorders and plays an important role in the diagnosis of epilepsy. We analyse and classify EEG signals using wavelets decomposition and support vector machines (SVM). In particular, we break the EEG waves into different time frames. Numerical experiment on a standard test data set demonstrates that the proposed algorithm can achieve high accuracy on the prediction of epilepsy even when short period of time duration is used.
\end{abstract}

Keywords

Electroencephalogram (EEG); Epilepsy Detection; Wavelets Decomposition; Data Classification; Support Vector Machines (SVM); Machine Learning

\section{Introduction}

Epilepsy is a serious chronic neurological disorder and it is characterized by successive unexpected seizures. Approximately one in every 100 persons will experience a seizure at some time in their life (Adeli et al., 2003 [1]). Electroencephalogram (EEG) is the electrical signal of brain which contains valuable information about its normal or epileptic (abnormal) activity (Agarwal et al., 1998 [2]). EEG signal has been widely used in clinical to investigate brain disorders and plays an important role in the diagnosis of epilepsy.

The EEG signal is often studied by decomposition into several frequencies (Ubeyli and Guler, 2007 [14]). Delta waves are associated with deep sleep in the frequency range of $0-4 \mathrm{~Hz}$ with high amplitude. Theta waves indicate the time of meditation, idling, or sometimes drowsiness, in the frequency range of 4-8 Hz. Alpha waves have a frequency range of $8-14 \mathrm{~Hz}$ which appear when the testee is relaxing or resting. Beta waves reside in the $13-30 \mathrm{~Hz}$ frequency band ranges and they are characteristic of the testee being alert or active, in particular when concentrating.

Gamma waves occur when sound or sight sensory is processed, in the frequency range of 30-100 Hz.

The discrete wavelet transform (DWT) is one of the most widely used tools in signal processing and many engineering and scientific applications, including epileptic seizure detection (Subasi, 2005 [11]). DWT captures transient features and analyzes the signal at different frequency bands, with different resolutions by decomposing the signal into a coarse approximation. DWT employs two sets of functions called scaling functions and wavelet functions, which are related to low-pass and high-pass filters, respectively. The decomposition of the signal into different frequency bands is obtained by consecutive high-pass and low-pass filtering of the time domain signal.

Quite a few methods have been applied to classifying EEG signals. For example, Guler and Ubeyli (2005) proposed an adaptive neuro-fuzzy inference system (ANFIS) model [8]. Wavelet transform is used for feature extraction and the ANFIS is trained with the backpropagation gradient descent method in combination with the least squares method. Five types of EEG signals were used as input patterns. Subasi (2007) introduced a double-loop ExpectationMaximization (EM) algorithm to the Mixture of experts (ME) ME network structure for detection of epileptic seizure [12]. EEG signals were decomposed into the frequency sub-bands using discrete wavelet trans- form (DWT). Then these sub-band frequencies were used as an input to a ME network with two discrete outputs: normal and epileptic. Zhong et al. (2008) applied Gaussian process (GP) classification to binary discrimination of motor imagery EEG data [16]. Band power (BP), the power of the signal in specific frequency bands, is used as features in the classification 
process. Omerhodzic et al. (2013) used the Discrete Wavelet Trans- form (DWT) with the Multi-Resolution Analysis (MRA) to decompose EEG signal at resolution levels of the components of the EEG signal. Then the neural network $(\mathrm{NN})$ is applied to identify the EEG types according to the percentage distribution of the energy features [10]. Li et al. (2014) proposed a multiple kernel learning support vector machine algorithm for the identification of EEG signals including mental and cognitive tasks [9]. Features include wavelet packet entropy and Granger causality and a gradient descent based optimization algorithm is investigated to classify EEG signals. Suguna Nanthini and Santhi (2014) used support vector machines (SVM) as the classifier and use gray level cooccurrence matrix (GLCM) to extract essential features from the EEG signal [13].

One of the most widely studied and publicly avail- able EEG data sets is from the manuscript by Andrzejak et al. (2001) [3]. The complete data set include five sets of data (denoted A-E), each contains 100 single-channel EEG segments. Each segment has N=4096 sampling points over 23.6 seconds. Many researchers have used this data set as a test bed for their work, e.g., Guler and Ubeyli (2005) [8], Subasi (2007) [12], Omerhodzic et al. (2013) [10], and Suguna Nanthini and Santhi (2014) [13], among others.

However, most existing work uses the entire segment over 23.6 seconds as a single sample in the classification. Samples of shorter time period will be more helpful and practically viable for epilepsy detection in real life. We use wave segments of different time durations in our study. Wavelet decomposition is used for feature extraction and SVM is used for classification. High (or higher than that of some existing work) accuracy is achieved.

\section{Materials and Methods}

In this study, we use the publicly available data set from Andrzejak et al. (2001) [3]. The complete data collection includes five sets (denoted A-E), each contains 100 single channel EEG segments of 23.6 seconds. These segments were selected and cut out from continuous multi-channel EEG recordings after visual inspection for artifacts, e.g., due to muscle activity or eye movements. Sets A and B consist of segments taken from surface EEG recordings that were carried out on five healthy volunteers using a standardized electrode placement scheme. Volunteers were relaxed in an awake state with eyes open (A) and eyes closed (B), respectively. Sets C, D, and E originated from EEG archive of pre-surgical diagnosis. EEGs from five patients were selected, all of whom had achieved complete seizure control after resection of one of the hippocampal formations, which was therefore correctly diagnosed to be the epileptogenic zone. Segments in set $\mathrm{D}$ were recorded from within the epileptogenic zone, and those in set $C$ from the hippocampal formation of the opposite hemisphere of the brain. While sets $C$ and $D$ contained only activity measured during seizure free intervals, set $\mathrm{E}$ only contained seizure activity. Here segments were selected from all recording sites exhibiting ictal activity. All EEG signals were recorded with the same 128channel amplifier system, using an average common reference. The data were digitized at 173.61 samples per second using 12 bit resolution. Band-pass filter settings were $0.53-40 \mathrm{~Hz}$ (12 dB/oct).

Three sets denoted A, C and E are used in this work. Each set contains 100 single channel EEG segments of 23.6 seconds duration at a sampling rate of $\mathrm{fs}=173.61 \mathrm{~Hz}$. Most existing work uses the entire segment of 23.6 second as a single sample in the classification process.

To make it more practically viable for epilepsy detection, we investigate the classification using EEG segments of shorter time duration. More specifically, we divide each segment of 23.4 seconds into 1/2,1/4, 1/5, 1/8, 1/10, 1/16, and 1/20, respectively, and the shorter EEG segments will be used as samples.

Wavelet transform (WT) is widely used for feature extraction in EEG signal analysis. The continuous wavelet transform $(\mathrm{CWT})$ of signal $\mathrm{f}(\mathrm{t})$ is defined as an integral:

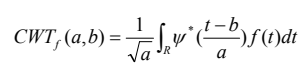

where $\psi$ is the wavelet function (mother wavelet), the parameter $a$ is the dilatation parameter (enables dilatation and compression of wavelet) and $b$ is the translation parameter. 
The discrete wavelet transform (DWT) is an adaptable signal processing tool that finds many engineering and scientific applications, including epileptic seizure detection. DWT captures transient features and analyzes the signal at different frequency bands, with different resolutions by decomposing the signal into a coarse approximation. Selection of appropriate wavelet and the number of decomposition levels are very important in signal analysis of using DWT. The number of decomposition levels is chosen based on the dominant frequency components of the signal. The levels are chosen such that those parts of the signal that correlate well with the frequencies required for classification of the signal are retained in the wavelet coefficients. In the present study, the Daubechies wavelet of order $4(\mathrm{db} 4)$ is used. Daubechies wavelet is one of the most commonly used set of discrete wavelet transforms which was formulated by the Belgian mathematician Ingrid Daubechies in 1988 [7]. This formulation is based on the use of recurrence relations to generate progressively finer discrete samplings of an implicit mother wavelet function; each resolution is twice that of the previous scale. The number of decomposition levels is chosen to be 5. Thus, the EEG signals were decomposed into the details D1-D5 and one final approximation, $A 5$.

For a given sample, we first apply the order 4 Daubechies wavelet of level 5 to obtain the de- compositions $D 1$ - D5. Then for each subband $D i, i=1,2,3,4,5$, we extract statistical features such as the mean, standard deviation, and entropy.

We use support vector machines (SVM) (see, e.g., Boser et al. (1992) [4], Cortes and Vapnik (1995)[6], and Vapnik (1998) [15]) for classification. In its simplest (linear) form, an SVM is a hyperplane that separates a set of positive examples from a set of negative examples with maximum margin. More precisely, given data points of the form $\left\{\left(y_{1}, x_{1}\right),\left(y_{2}, x_{2}\right), \ldots,\left(y l, x_{l}\right)\right\}$, where the $y_{i}$ is either 1 or -1 , a constant denoting the class to which the point $x_{i}$ belongs. Each $x_{i}$ is an $\mathrm{n}$-dimensional real vector. We can view this as training data, which denotes the correct classification that we would like the SVM to eventually distinguish, by means of the dividing (or separating) hyperplane. The hyperplane takes the form $w \cdot x-b=0$. The coefficient vector $w$ points perpendicular to the separating hyperplane. The two-class SVM can be adapted for multi-class classification as well.

\section{Results and Discussion}

Three sets denoted A (healthy, open eye), C (prior to a seizure), and E (during a seizure) give us three classes. Each set contains 100 single channel EEG segments of 23.6 seconds time duration.

If an entire EEG segment is used as one sample, we would have 300 samples. Clearly it is more practically viable for epilepsy detection if shorter time durations are used. When each segment is divided into $1 / 2,1 / 4,1 / 5,1 / 8,1 / 10,1 / 16$, and $1 / 20$, the time durations will be roughly $11.8,5.9,4.72,2.95,2.36,1.475$, and 1.18 seconds, and the number of samples will be 300, 1200, 1500, 2400, 3000, 4800, and 6000, respectively.

Our numerical experiments were carried out using Matlab, and the Matlab functions wavedec, detcoef, mean, std, wentropy were used for wavelet decomposition, wavelet coefficient calculation, and feature extraction.

The SVM package LIBSVM [5] was used in our experiments. It has the capability for multi-class classification. We tried different kernels. It turned out that even the simplest linear kernel worked very well: high accuracy was achieved for all cases. In addition, the classification accuracy was not sensitive to the error-tolerance constant $\mathrm{C}$. In our experiments, the value $C=100$ was used for all time durations. We used $50 \%$ samples for training and $50 \%$ samples for testing. Since the three classes are well balanced, the accuracy for each class will be a good measure for classification quality. The classification result is summarized in the following table.

The experiment result shows that the wavelet decomposition with the selected statistical features, together with the support vector machine classifier, works very well to separate the three classes, even when the time duration of the EEG signal is reduced to about one second. It is interesting to note that when the time duration is shortened, the EEG signals during seizure remain very distinctive from the other two. However, the distinction between the healthy group and the prior to seizure group will get more blurred when the time frames are reduced. 
Table 1: Classification Accuracy for Different Classes and Time Durations

\begin{tabular}{|r|c|c|c|c|}
\hline EEG (sec) & All Sets & Set A & Set C & Set E \\
\hline 23.6000 & 96.6667 & 98.0000 & 96.0000 & 96.0000 \\
\hline 11.8000 & 98.0000 & 98.0000 & 97.0000 & 99.0000 \\
\hline 5.9000 & 96.6667 & 97.0000 & 93.5000 & 99.5000 \\
\hline 4.7200 & 97.0667 & 98.4000 & 94.4000 & 98.4000 \\
\hline 2.9500 & 95.0833 & 92.0000 & 94.0000 & 99.2500 \\
\hline 2.3600 & 96.7333 & 96.0000 & 95.0000 & 99.2000 \\
\hline 1.4750 & 94.7500 & 93.7500 & 91.6250 & 98.8750 \\
\hline 1.1800 & 93.6000 & 91.5000 & 90.6000 & 98.7000 \\
\hline
\end{tabular}

\section{Conclusions}

Using a standard data collection, we investigated classification quality when the time duration of EEG signals is reduced to a short period. The high accuracy of the experiment result demonstrates that the proposed procedure might be a viable tool for epilepsy detection, even clinically. More extensive study and clinical trials will be needed to confirm that.

\section{REFERENCES}

[1] H. Adeli, Z. Zhou, and N. Dadmehr, Analysis of EEG records in an epileptic patient using wavelet transform, Journal of Neuro- science Methods, 123 (2003) 69-8

[2] R. Agarwal, J. Gotman, D. Flanagan, and B. Rosenblatt, Automatic EEG analysis during long-term monitoring in the ICU, Electroencephalography and Clinical Neurophysiology, 107(1) (1998) 44-58.

[3] R.G. Andrzejak, K. Lehnertz, F. Mormann,C. Rieke, P. David, and C.E. Elger, Indications of nonlinear deterministic and finite dimensional structures in time series of brain electrical activity: Dependence on recording region and brain state, Physical Review E, 64 (2001) 061907.

[4] B.E. Boser, I.M. Guyon, and V. Vapnik, A Training Algorithm for Optimal Margin Classifiers, Fifth Annual Workshop on Computational Learning Theory, ACM, 1992.

[5] C.C. Chang and C.J. Lin, LIBSVM: a library for support vector machines, (software available at http ://www.csie.ntu.edu.tw/ cjlin/libsvm),2001.

[6] C. Cortes and V. Vapnik, Support Vector Networks, Machine Learning, 20, (1995) pp.273-297.

[7] I. Daubechies, Orthonormal Bases of Compactly Supported Wavelets, Communications on Pure and Applied Mathematics, Vol. 41, No.7, (1988), pp. 909-996.

[8] I. Guler and E.D. Ubeyli, Adaptive neuro-fuzzy inference system for classification of EEG signals using wavelet coefficients, Journal of Neuroscience Methods, Volume 148, Issue 2, (2005), Pages 113-121.

[9] X. Li, X. Chen, Y. Yan, W. Wei, and Z.J. Wang, Classification of EEG Signals Using a Multiple Kernel Learning Support Vector Machine, Sensors, 14 (2014) 12784-12802.

[10] I. Omerhodzic, S. Avdakovic, A. Nuhanovic, and K. Dizdarevic, Energy Distribution of EEG Signals: EEG Signal WaveletNeural Network Classifier, World Academy of Science, Engineering and Technology, 61 (2013) 1190-1195.

[11] A. Subasi, Epileptic seizure detection using dynamic wavelet network, Expert Systems with Applications, 29 (2005), 343355.

[12] A. Subasi, EEG signal classification using wavelet feature extraction and a mixture of expert model, Expert Systems with Applications, 32 (2007) 1084-1093.

[13] B. Suguna Nanthini and B. Santhi, Seizure Detection using SVM Classifier on EEG Signal, Journal of Applied Sciences, 14 (2014) 1658-1661. 
[14] E.D. Ubeyli and I. Guler, Features extracted by eigenvector methods for detecting variability of EEG signals, Pattern Recognition Letters 28(5):592-603, 2007.

[15] V. Vapnik, Statistical Learning Theory, John Wiley, New York, 1998.

[16] M. Zhong, F. Lotte, M. Girolami, and A. Lecuyer, Classifying EEG for Brain Computer Interfaces Using Gaussian Process, Pattern Recognition Letters, Volume 29, Is- sue 3 (2008), Pages 354-359. 